\title{
Retrospective analysis of canine gallbladder contents in biliary sludge and gallbladder mucoceles
}

\author{
Shinya MIZUTANI'), Shidow TORISU ${ }^{1) *}$, Yasuyuki KANEKO'), \\ Shushi YAMAMOTO ${ }^{1)}$, Shinsuke FUJIMOTO ${ }^{1,2)}$, Benedict Huai Ern ONG ${ }^{1)}$ and \\ Kiyokazu NAGANOBU ${ }^{1)}$ \\ 1)University of Miyazaki Veterinary Teaching Hospital, 1-1 Gakuen Kibana-dai Nishi, Miyazaki City, Miyazaki \\ 889-2192, Japan \\ ${ }^{2)}$ Ozu Animal Clinic, 317 Muro, Ozu Town, Kikuchi District, Kumamoto 869-1235, Japan
}

J. Vet. Med. Sci.

79(2): 366-374, 2017

doi: 10.1292/jvms.16-0562

Received: 1 November 2016

Accepted: 18 November 2016

Published online in J-STAGE:

17 December 2016
ABSTRACT. The pathophysiology of canine gallbladder diseases, including biliary sludge, gallbladder mucoceles and gallstones, is poorly understood. This study aimed to evaluate the component of gallbladder contents and bacterial infection of the gallbladder in order to elucidate the pathophysiology of biliary sludge and gallbladder mucoceles. A total of 43 samples of canine gallbladder contents (biliary sludge, 21 and gallbladder mucoceles, 22) were subjected to component analysis by infrared spectroscopy, and the resultant infrared spectra were compared with that of swine mucin. Of the 43 samples, 41 were also evaluated by aerobic and anaerobic bacterial culture. The contents of 20 (95.2\%) biliary sludge and $22(100 \%)$ gallbladder mucocele samples exhibited similar infrared spectra as swine mucin. Although biliary sludge and gallbladder mucocele contents exhibited similar infrared spectra, one sample of biliary sludge (4.8\%) was determined to be composed of proteins. The rate of bacterial infection of the gallbladder was $10.0 \%$ for biliary sludge and $14.3 \%$ for gallbladder mucoceles. Almost all of the identified bacterial species were intestinal flora. These results indicate that the principal components of gallbladder contents in both gallbladder mucoceles and biliary sludge are mucins and that both pathophysiologies exhibit low rates of bacterial infection of the gallbladder. Therefore, it is possible that gallbladder mucoceles and biliary sludge have the same pathophysiology, and, rather than being independent diseases, they could possibly represent a continuous disease. Thus, biliary sludge could be considered as the stage preceding the appearance of gallbladder mucoceles.

KEY WORDS: biliary sludge, dog, gallbladder mucocele, infrared spectroscopy, mucin

Canine gallbladder diseases including biliary sludge, gallbladder mucoceles and gallstones are being commonly diagnosed in dogs, because of the increase in the use of ultrasonography in recent times. Biliary sludge is a disease where a sludge-like substance accumulates in the gallbladder. Abdominal ultrasonography findings of gallbladder contents in biliary sludge reveal the presence of hyperechogenic sludge. Biliary sludge occurs more commonly in older dogs. Its pathophysiology is poorly understood, and it is often treated with internal medicine, with a low-fat diet and medications, such as ursodeoxycholic acid, S-adenosylmethionine and other drugs $[1,3,4,8,11,17,25]$. However, severe biliary sludge, in which the gallbladder contents almost completely fill the gallbladder, might require cholecystectomy.

Gallbladder mucocele is characterized by the excessive secretion of mucin from gallbladder epithelium, which leads to progressive accumulation of highly-viscid bile in the gallbladder, resulting in varying degrees of extrahepatic bile duct obstruction [2]. Progressive expansion of the gallbladder due to a gallbladder mucocele might lead to ischemic necrosis of the gallbladder wall and/or bile peritonitis, because of a ruptured gallbladder. Gallbladder mucoceles causing extrahepatic bile duct obstruction are treated by cholecystectomy. However, the perioperative mortality rate for gallbladder mucoceles is high $(21.7-40.0 \%)$ [5, 20]. A better understanding of the pathophysiology of gallbladder mucoceles could help reduce this risk.

Mucins, which play a major role in the development of gallbladder mucoceles, are a family of polysaccharides secreted by mucosal epithelial cells of the gallbladder, stomach, intestines and other organs. Mucins act as surfactants and play a mucoprotective role by preventing self-digestion of mucosal epithelium by digestive juices $[6,10,12]$. Abdominal ultrasonography

*Correspondence to: Torisu, S., University of Miyazaki Veterinary Teaching Hospital, 1-1 Gakuen Kibana-dai Nishi, Miyazaki City, Miyazaki 889-2192, Japan. e-mail: torisu@cc.miyazaki-u.ac.jp

(C2017 The Japanese Society of Veterinary Science

This is an open-access article distributed under the terms of the Creative Commons Attribution Non-Commercial No Derivatives (by-ncnd) License <http://creativecommons.org/licenses/by-nc-nd/4.0/>. 

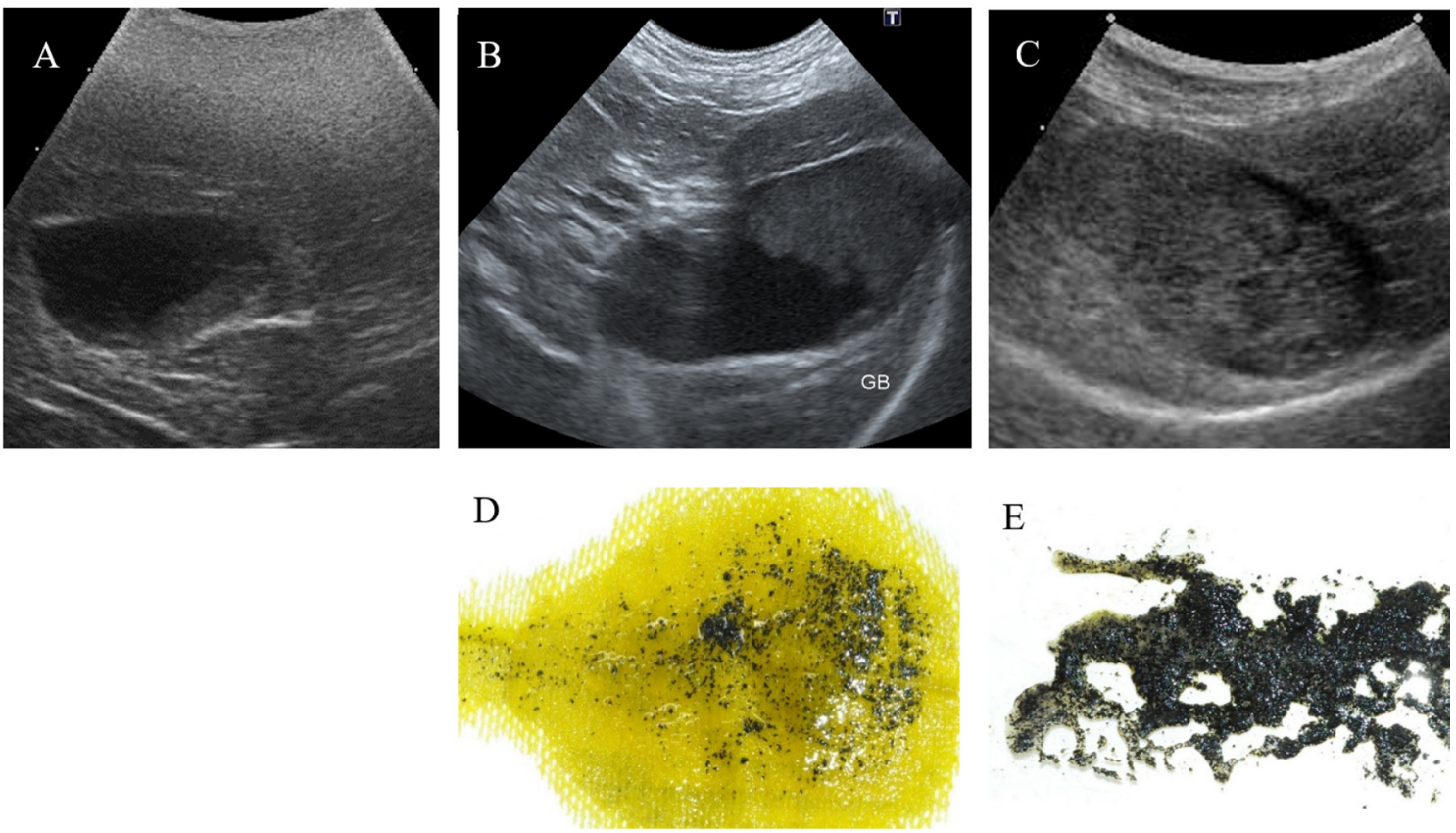

Fig. 1. Ultrasonography images and gallbladder content findings of biliary sludge. Biliary sludge presents as hyperechogenic accumulated matter in the direction of gravity on ultrasonography images — mild* (A), moderate* (B) and severe* (C). Gallbladder contents in moderate biliary sludge (D) have a black sand-like appearance, while those in severe biliary sludge (E) have a black sludge-like appearance. *Abdominal ultrasonography was performed in the standing position. Biliary sludge was classified according to its rate of retention within the gallbladder as mild $(\leq 25 \%)$, moderate $(25-75 \%)$ or severe $(\geq 75 \%)$.

is widely used for the diagnosis of gallbladder mucoceles, which present as a characteristic "kiwi fruit" pattern in the gallbladder [5]. Biliary sludge and gallbladder mucoceles are generally thought of as independent diseases. However, with abdominal ultrasonography being commonly used as a diagnostic modality in veterinary medicine, these two diseases have been reported to sometimes exhibit similar findings. In fact, a previous report has noted that, in certain cases, there is no clear distinction between the findings of severe biliary sludge and gallbladder mucoceles, which makes their differentiation challenging [23].

Although there have been a few reports on the analysis of canine gallstone contents and bacterial infection of the gallbladder, no study till date has performed component analysis of gallbladder contents in biliary sludge. To the best of our knowledge, there are no reports on component analysis of gallbladder contents in gallbladder mucoceles either. Additionally, few studies have reported on bacterial infection of the gallbladder in biliary sludge and gallbladder mucoceles [20]. Therefore, for the purpose of elucidating the pathophysiologies of the two diseases, this study aimed to perform component analysis of gallbladder contents in biliary sludge and gallbladder mucoceles by infrared (IR) spectroscopy [9, 13-16, 26], which is widely used for component analysis of gallstones and bladder stones, and to evaluate bacterial infection of the gallbladder.

\section{MATERIALS AND METHODS}

This retrospective study included 40 dogs that were diagnosed with biliary sludge and gallbladder mucoceles, and had their gallbladder contents collected during surgery or autopsy at the University of Miyazaki Veterinary Teaching Hospital, Miyazaki, Japan, between May 2012 and July 2016. Component analysis was performed using 43 samples of gallbladder contents from 40 dogs diagnosed with biliary sludge or gallbladder mucoceles - for three samples, the same animal was re-examined over the course of follow-up. Samples that were diagnosed by autopsy were from dogs that either died of natural causes or were euthanized for reasons other than gallbladder diseases. Euthanization was conducted as below: first, dogs were administered with pentobarbital sodium (50 mg/kg, IV; Somnopentyl, Kyoritsu Seiyaku Corp., Tokyo, Japan). Five min later, potassium chloride (2 mEq/kg, IV; KCL Corrective Injection $1 \mathrm{mEq} / \mathrm{m} l$, Otsuka Pharmaceutical Factory, Inc., Tokushima, Japan) was then administered.

Biliary sludge and gallbladder mucoceles were diagnosed by gross examination during surgery or autopsy. Biliary sludge was diagnosed when the gallbladder contents presented a sludge-like appearance. Gallbladder mucoceles were diagnosed when the gallbladder appeared to be completely filled with a jelly-like substance (Figs. 1 and 2). Biliary sludge was classified according to severity based on the content retention rate relative to size of the gallbladder determined by ultrasonography, as follows: mild ( $\leq 25 \%$ retention), moderate $(25-75 \%$ retention) or severe $(\geq 75 \%$ retention; Fig. 1$)$.

Of the 40 dogs included in the present study, 18 (21 samples) exhibited biliary sludge, and 22 (22 samples) exhibited gallbladder mucoceles. Dogs included in this study were of the following breeds: Miniature Dachshund, Chihuahua and Toy Poodle (n=6, each); Miniature Schnauzer ( $n=4)$; Pug and mixed breed ( $n=3$, each); American Cocker Spaniel, Shetland Sheepdog, Yorkshire 

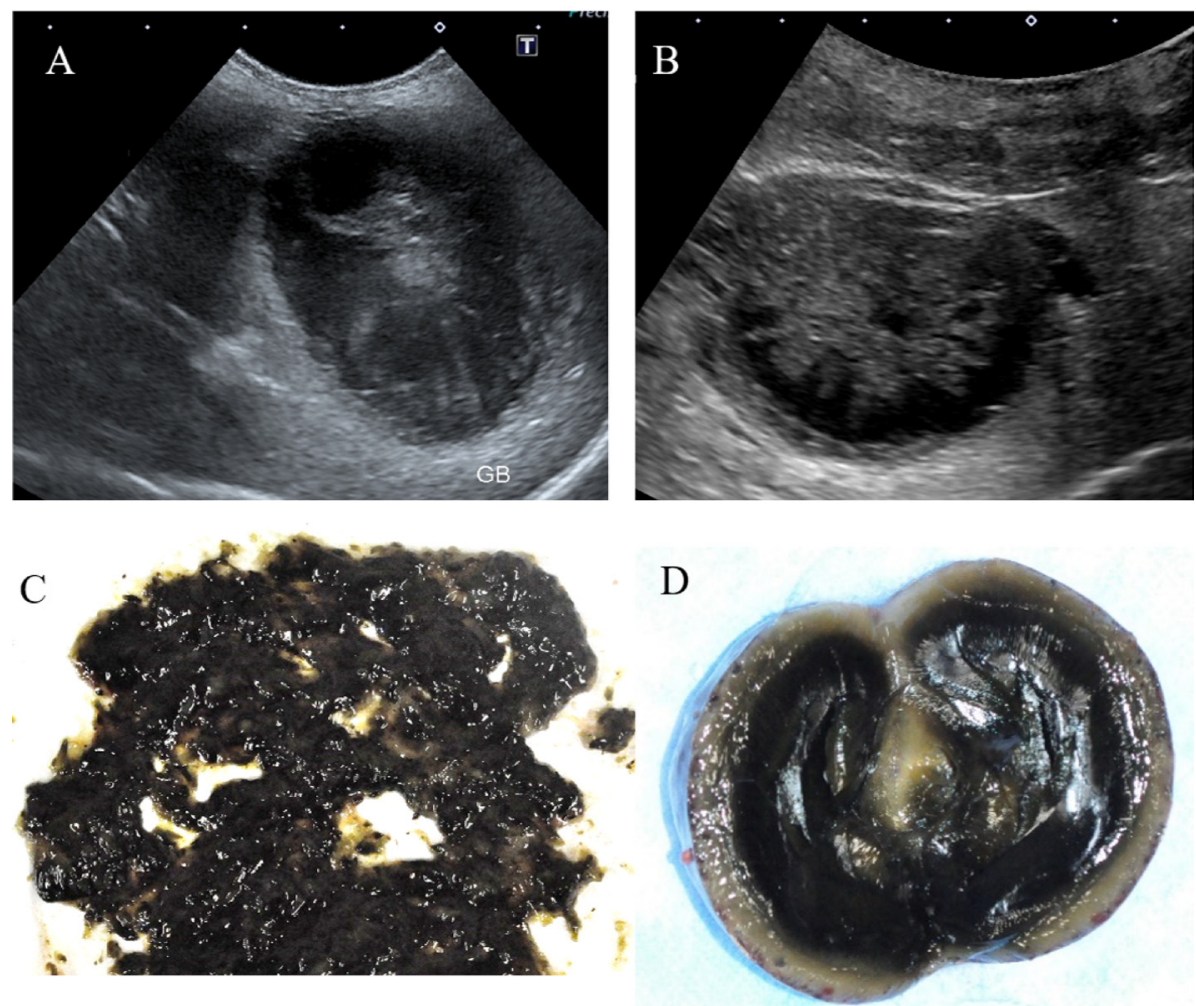

Fig. 2. Ultrasonography images and gallbladder content findings of gallbladder mucoceles. Gallbladder mucoceles characteristically present as hyperechogenic accumulated material in a "kiwi fruit" pattern within the gallbladder on abdominal ultrasonography images (A and B). However, gallbladder contents in gallbladder mucoceles present varied appearances; in the present study, while gallbladder contents in a few mucoceles presented a black and jelly-like appearance (C), those in other mucoceles presented as an indurated, jelly-like material radiating to the margin (D).

Terrier, Beagle, French Bulldog and Doberman (n=2, each) and Pomeranian, Shiba Inu and Papillon ( $\mathrm{n}=1$, each). The median age of the 40 dogs (43 samples) included in the present study (male, 5; castrated male, 11; female, 7; and spayed female, 20) was 9 years (range, 1-15 years, including one dog of unknown age), and the median weight was $5.2 \mathrm{~kg}(\mathrm{range}, 1.9-29.0 \mathrm{~kg}$ ). All dogs were examined with the owners' consent having been obtained prior to surgical treatment (cholecystectomy, 25 dogs and gallbladder irrigation, 15 dogs) or autopsy ( 3 dogs). Gallbladder irrigation was performed by making a small incision on the gallbladder wall, followed by aspiration of contents in the lumen of the gallbladder and irrigation and cleaning up of the inside of the gallbladder with saline.

For component analysis, 43 canine gallbladder content samples with bile (biliary sludge, 21 and gallbladder mucoceles, 22) were first dried well and then prepared as potassium bromide (KBr) pellets for evaluation by IR spectroscopy (IR spectroscopy FT/IR410, JASCO Corp., Tokyo, Japan.). In a previous study [22] on the IR spectroscopy of ovary-derived mucins, bovine submaxillary gland-derived mucins were used as reference. Therefore, mucins derived from swine stomach (swine mucin; purity $\geq 80 \%$, Wako Pure Chemical Industries, Ltd., Tokyo, Japan.) were used to establish the reference IR spectrum for mucins in this study. The IR spectra of biliary sludge and gallbladder mucoceles contents were compared with that of swine mucin in order to determine the composition of the former.

Of the 43 canine gallbladder content samples, 41 (biliary sludge, 20 and gallbladder mucoceles, 21) were evaluated by bacterial culture. The animals were administered antimicrobials after collection of gallbladder contents, but not within $12 \mathrm{hr}$ prior to collection. Bacterial cultures of gallbladder contents were established by aseptically swabbing the inside of the gallbladder with a sterile cotton swab and inoculating the samples onto bacterial media for aerobic and anaerobic culture, followed by bacterial identification.

\section{RESULTS}

\section{Severity classification of canine biliary sludge}

As determined by ultrasonographic findings, there was no mild case, moderate was 10 cases, with all samples exhibiting biliary sludge retention rates $\geq 50 \%$, and severe was 11 cases. 


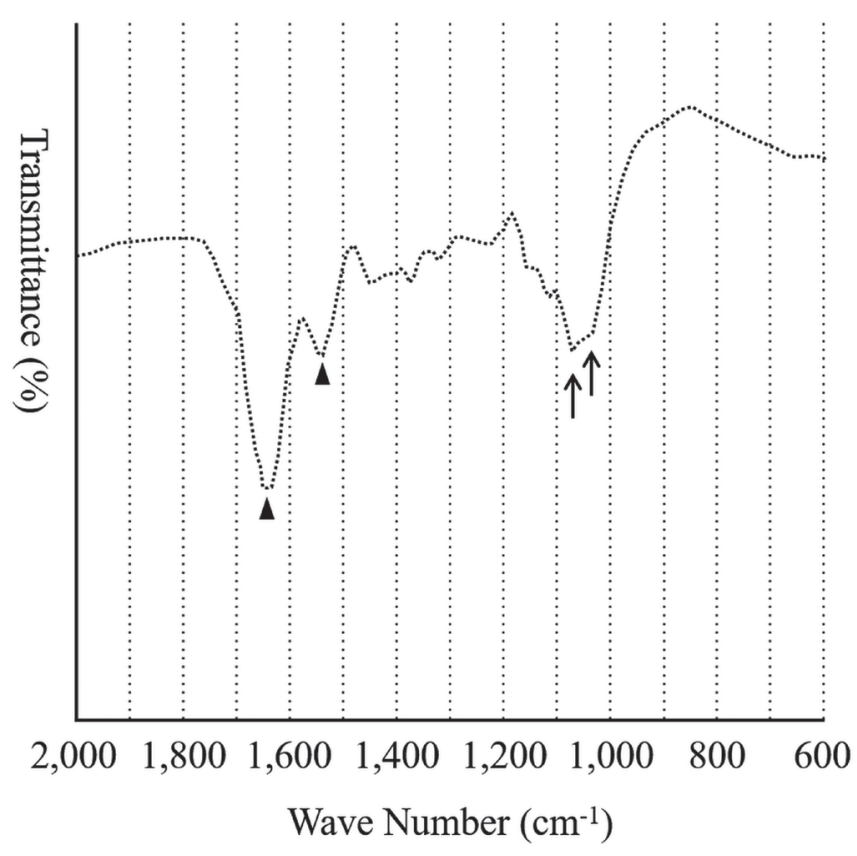

Fig. 3. IR spectrum of swine mucin. Swine mucin exhibits characteristic absorption bands of proteins (amide bands near 1,650 and $1,550 \mathrm{~cm}^{-1}$; indicated by arrowheads) and sugar chains (glycosylation bands near 1,070 and $1,050 \mathrm{~cm}^{-1}$; indicated by arrows).

\section{IR spectrum of swine mucin}

The IR spectrum of swine mucin corresponded to proteins, with amide bands near 1,650 and 1,550 $\mathrm{cm}^{-1}$, and polysaccharides, with spectral characteristics of sugar chains (glycosylation bands) near 1,070 and 1,050 $\mathrm{cm}^{-1}$ (Fig. 3) [22]. This IR spectrum was used as the reference for identification of mucins in gallbladder contents.

\section{IR spectra and component analysis of gallbladder contents}

Of the 21 samples of gallbladder contents of dogs with biliary sludge, $20(95.2 \%)$ - ten samples each of moderate and severe biliary sludge - exhibited IR spectra coinciding with amide and glycosylation bands similar to those observed with swine mucin and were thus identified as being composed of mucins (Fig. 4). The IR spectrum of the one remaining sample (4.8\%), which was from a dog with severe biliary sludge, did not exhibit clear glycosylation bands and instead presented only amide bands; this sample was, therefore, identified as being composed of proteins (Fig. 4).

All 22 samples of gallbladder contents of dogs with gallbladder mucoceles exhibited IR spectra coinciding with amide and glycosylation bands similar to those observed with swine mucin and were thus identified as mucins (Fig. 5). The IR spectra of biliary sludge and gallbladder mucoceles contents were found to be similar.

\section{IR spectra of gallbladder contents of the same dog at multiple time points}

Three of the dogs with biliary sludge received gallbladder irrigation, followed by 1-2 years of follow-up, at the end of which, progressively accumulated gallbladder contents were again collected by gallbladder irrigation. Of the three dogs, one underwent cholecystectomy for extrahepatic bile duct obstruction due to re-accumulation of gallbladder contents about 1 year and 8 months after the second gallbladder irrigation. Unlike the other two dogs, the gallbladder contents of this dog at the time of the cholecystectomy had clearly transitioned from sludge-like to jelly-like appearance. Therefore, the dog was diagnosed with a gallbladder mucocele rather than biliary sludge based on the findings of analysis of gallbladder contents from the third collection (Fig. 6). The gallbladder contents of this dog exhibited nearly similar IR spectra at all three collections and were thus identified each time as being composed of mucins (Fig. 7). The gallbladder contents of the remaining two dogs presented a sludge-like appearance at the first and second collections, based on which the dogs were diagnosed with biliary sludge. The gallbladder contents were identified as being composed of mucins based on their IR spectroscopic findings.

\section{Bacterial infection of the gallbladder}

Of the 20 biliary sludge samples, $2(10.0 \%)$ exhibited bacterial infection. The infective bacterial species were identified to be Escherichia coli and Serratia marcescens in one sample each. Of the 21 gallbladder mucocele samples, 3 (14.3\%) exhibited bacterial infection. The infective bacterial species were identified to be Escherichia coli in two samples and Enterococcus spp. in one sample. 


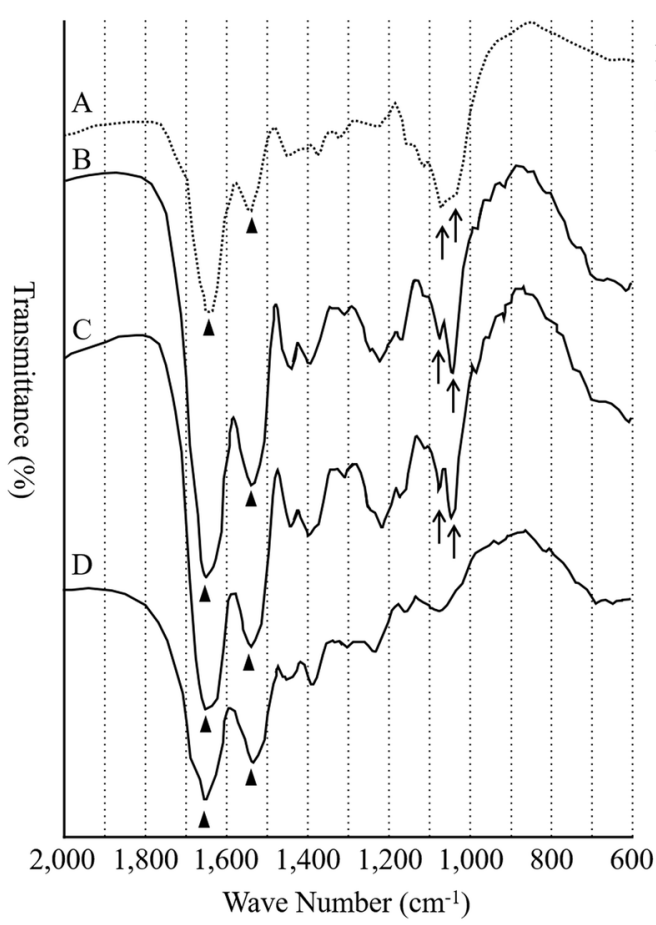

A: swine mucin

B: biliary sludge (severe)

C: biliary sludge (moderate)

D: biliary sludge (severe)

Fig. 4. IR spectra of swine mucin and biliary sludge. IR spectra of swine mucin (A; dotted line) and severe (B; solid line) and moderate (C; solid line) biliary sludge*. IR spectra of severe biliary sludge that was identified as proteins (D; solid line). Moderate (B) and severe (C) biliary sludge both exhibited amide bands (near 1,650 and 1,550 $\mathrm{cm}^{-1}$; indicated by arrowheads) and glycosylation bands (near 1,070 and $1,050 \mathrm{~cm}^{-1}$; indicated by arrows), with the exception of one sample (D) of severe biliary sludge, where only amide bands were observed. *IR spectra of only representative samples are presented in B and C; B (n=9) and C $(n=10)$ exhibited similar spectra.

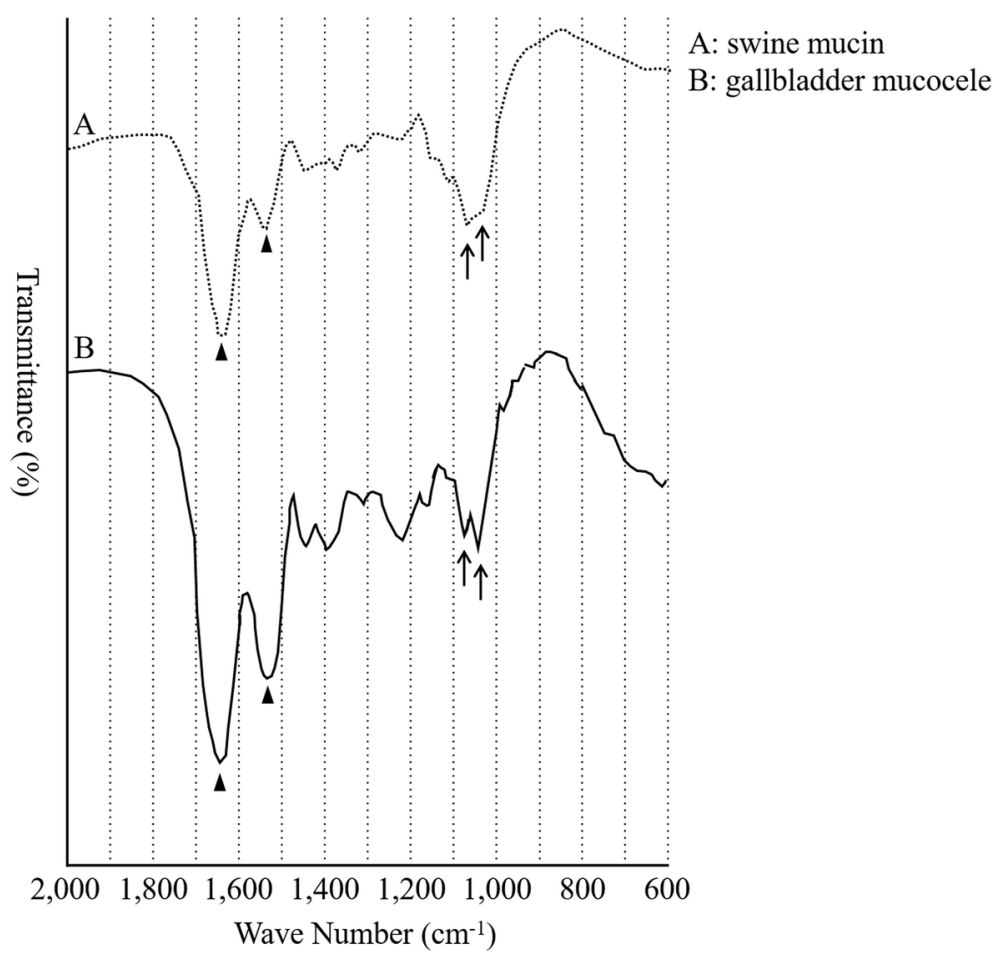

Fig. 5. IR spectra of swine mucin and gallbladder mucoceles. IR spectra of swine mucin (A; dotted line) and gallbladder mucoceles* (B; solid line). Gallbladder mucoceles (B) exhibited amide bands (near 1,650 and 1,550 $\mathrm{cm}^{-1}$; indicated by arrowheads) and glycosylation bands (near 1,070 and $1,050 \mathrm{~cm}^{-1}$; indicated by arrows). ${ }^{*}$ IR spectrum of only a representative sample is presented in B. The gallbladder contents of all gallbladder mucoceles $(n=21)$ exhibited similar spectra. 

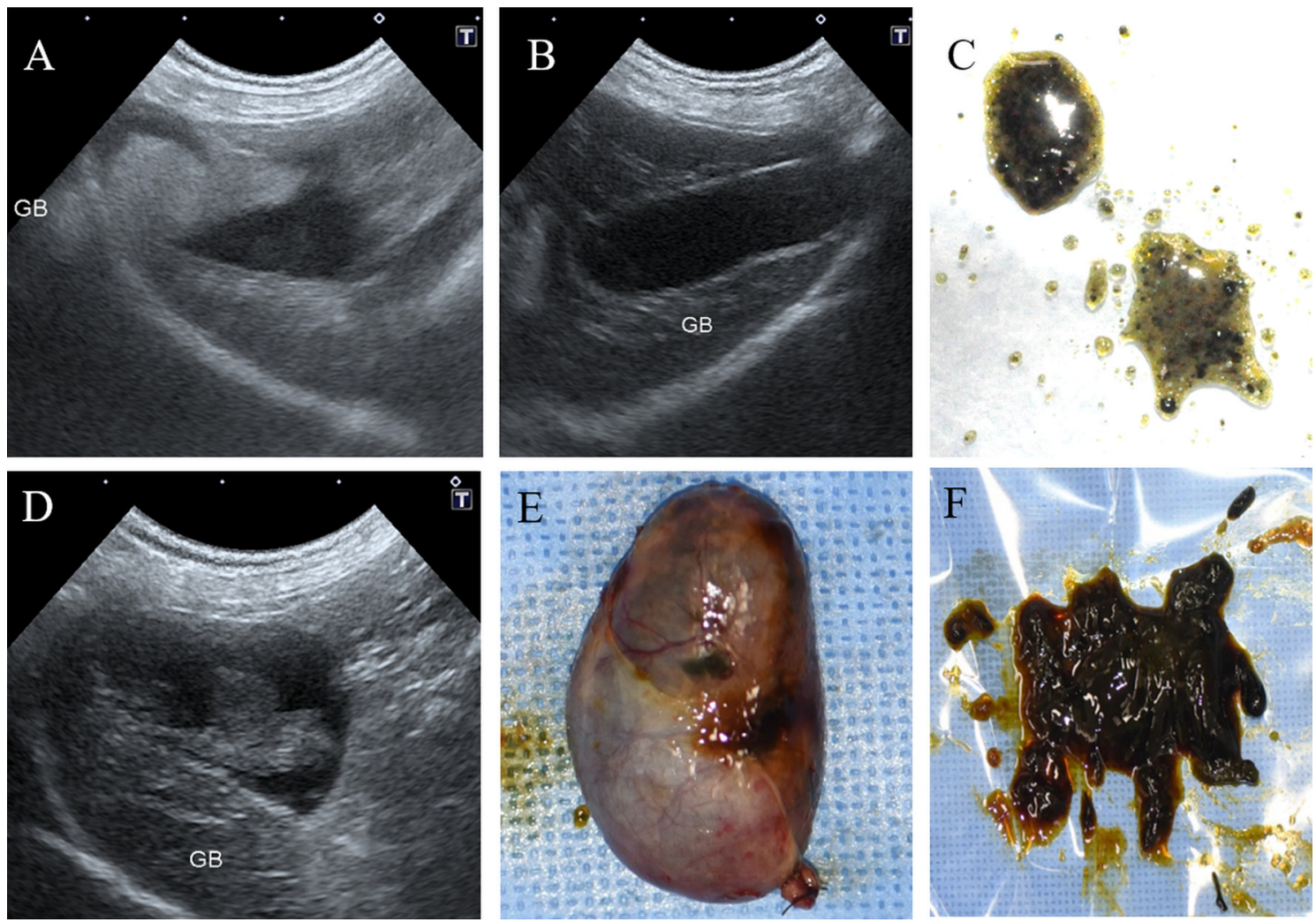

Fig. 6. Ultrasonography and gallbladder content findings of the same dog at different time points. Ultrasonography findings at 1 year and 7 months after first collection (A) and 10 days after second collection (B) indicated biliary sludge. Gallbladder contents of both first and second collections were similar and determined to be biliary sludge (C). Ultrasonography findings at 1 year and 8 months after second collection when extrahepatic duct obstruction occurred (D). Findings of content analysis of the excised gallbladder (E). Gallbladder content findings at third collection indicated a gallbladder mucocele (F). For treatment of biliary sludge, the inside of the gallbladder was cleaned with saline, and all of the sludge-like content (C) was collected (B). However, biliary sludge recurred and accumulated progressively (D). During the interval of 1 year and 7 months between the initial collection of biliary sludge and second collection of gallbladder contents, the dog exhibited no clinical symptoms of extrahepatic bile duct obstruction, and its recovery was uneventful. However, upon recurrence of biliary sludge, the patient received additional follow-up for 1 year and 8 months. To resolve the extrahepatic bile duct obstruction observed during follow-up (D), the patient underwent cholecystectomy (E). The contents of the excised gallbladder had changed in appearance from sludge (C) to jelly-like consistency (F), leading to the diagnosis of gallbladder mucocele.

\section{DISCUSSION}

Canine gallbladder diseases - especially gallbladder mucoceles - are caused by excessive mucin secretion [2]. However, to our knowledge, there are no reports regarding component analysis of actual contents from gallbladder mucoceles. A previous study performed IR spectroscopic analysis of human ovary-derived mucins with the IR spectrum of bovine submaxillary gland-derived mucins as reference [22]. Therefore, in the present study, the IR spectrum of swine mucin was employed as the reference for comparative evaluation of IR spectra of biliary sludge and gallbladder mucocele samples. Nearly all of the gallbladder content samples of dogs with biliary sludge (95.2\%) exhibited similar IR spectra as swine mucin, irrespective of the severity of biliary sludge, which ranged from moderate (50-75\% biliary sludge) to severe (Fig. 4). In case of gallbladder mucocele contents, all samples exhibited similar IR spectra as swine mucin (Fig. 5). These findings indicated that the principal components of gallbladder contents in both biliary sludge and gallbladder mucoceles were mucins, which suggested the possibility that mucins were involved in the pathogenesis of not only gallbladder mucoceles but also biliary sludge. However, in the IR spectra of Fig. 7, it was observed that there were some changes between biliary sludge (A and B) and gallbladder mucocele (C). In particular, these changes were observed near 1,460, 1,375 and 1,055 $\mathrm{cm}^{-1}$ in Fig. 7 (C). These were characteristic IR spectra showing cholesterol [14]. It was suggested that these IR spectra were possible to have been affected by cholesterol because cholesterol was contained in the bile. Therefore, it was possible that amount of cholesterol in the gallbladder content was increased with varying from biliary sludge to gallbladder mucocele in this case. Although these characteristic IR spectra showing cholesterol were observed in some of gallbladder mucocele cases, not in all gallbladder mucocele cases. Therefore, since all gallbladder mucocele samples in this study showed amide bands and glycosylation bands, it was considered that the principal component of gallbladder mucocele was mucin.

In present study, the gallbladder contents of one of the dogs with biliary sludge were identified as being composed of proteins 


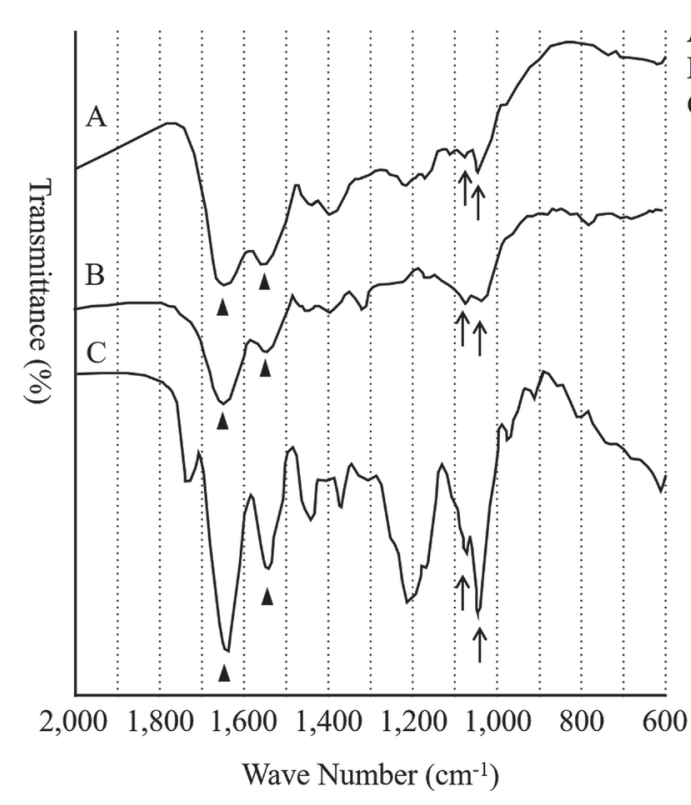

A: biliary sludge (first collection)

B: biliary sludge (second collection)

C: gallbladder mucocele (third collection)

Fig. 7. IR spectra of gallbladder contents of the same dog at different time points. IR spectra of gallbladder contents from the first (A) and second (B) collections, both of which were determined to be biliary sludge. IR spectra of gallbladder contents from the third collection (C), which was determined to be from a gallbladder mucocele. Gallbladder contents from the first, second and third collections exhibited similar IR spectra; both amide bands (near 1,650 and 1,550 $\mathrm{cm}^{-1}$; indicated by arrowheads) and glycosylation bands (near 1,070 and 1,050 $\mathrm{cm}^{-1}$; indicated by arrows) were observed, and the contents were identified as mucins.

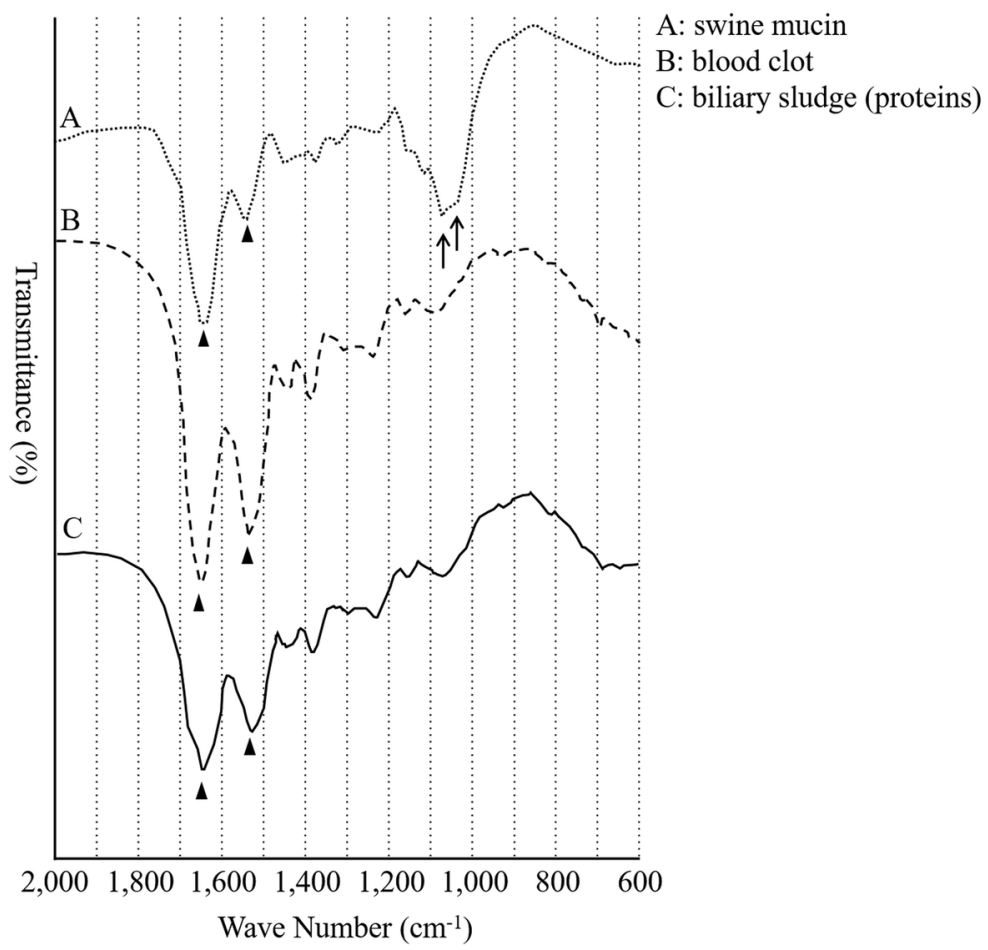

Fig. 8. IR spectra of swine mucin, blood clot and biliary sludge composed of proteins. IR spectra of swine mucin (A; dotted line) and a blood clot from a healthy $\operatorname{dog}(\mathrm{B}$; dashed line). Gallbladder contents of biliary sludge determined as being composed of proteins (C; solid line). The blood clot exhibited only amide bands in its IR spectrum and was, therefore, determined as being composed of proteins. The IR spectra of the blood clot and the biliary sludge determined as being composed of proteins were similar. Arrowheads indicate amide bands (near 1,650 and $\left.1,550 \mathrm{~cm}^{-1}\right)$; arrows indicate glycosylation bands (near 1,070 and $1,050 \mathrm{~cm}^{-1}$ ). 
(Fig. 4). The gallbladder in this case had presented extensive bleeding upon histopathological examination, which indicated the possibility that gallbladder bleeding affected the composition of the biliary sludge contents. In order to verify these hypothesis, the IR spectrum of a clot of peripheral venous blood from a healthy dog was compared with that of the gallbladder contents in this case. The IR spectrum of the blood clot exhibited amide bands but no glycosylation bands, and therefore, the blood clot was identified as being composed of proteins. The IR spectrum of the proteinaceous biliary sludge sample was found to be similar to that of the blood clot (Fig. 8), which may be attributable to the mixing of biliary sludge with plasma proteins from blood because of gallbladder bleeding. These findings suggest that, in cases where histopathological findings reveal gallbladder bleeding, the composition of gallbladder contents might be different from mucin.

Both biliary sludge and gallbladder mucocele samples exhibited a low incidence of bacterial infection (10.0 and $14.3 \%$, respectively). A previous study reported a $13.5 \%$ rate of bacterial infection in gallbladder mucoceles [20], which corresponds with the findings of the present study. Several bacterial species identified in gallbladder infections are among those that constitute normal intestinal flora [7]. Therefore, it is possible that the cases of gallbladder infection observed in the present study were due to ascending infection from the duodenum.

The findings of component analysis of gallbladder contents from canine biliary sludge and gallbladder mucoceles in the present study have demonstrated the previously unreported fact that mucins are the principle components of gallbladder content in both pathophysiologies. There were no differences in gallbladder composition among biliary sludge samples of various severities. Additionally, the low rates of bacterial infection in both biliary sludge and gallbladder mucocele samples indicated that these two disease differ in pathophysiology from gallstones [2].

From the results of this study, it was considered that the pathophysiology of canine biliary sludge may be occurred by progressive accumulation of mucin in the gallbladder. In one of the dogs included in the present study, gallbladder disease progressed from biliary sludge to gallbladder mucocele (Fig. 6). These findings suggest not only that biliary sludge and gallbladder mucoceles share a pathophysiology involving the progressive accumulation of mucins, but also that biliary sludge possibly represents the stage preceding gallbladder mucoceles. However, the factors responsible for the transition of gallbladder contents from sludge to jelly-like appearance in the present study are not clear. As a criterion for differentiating between the sludge and jelly-like appearances of gallbladder contents, in the present study, a resected gallbladder with contents that were semisolid, low in liquid content and appeared molded to the gallbladder was diagnosed as a gallbladder mucocele. The water content of gallbladder contents might be one of the factors responsible for the transition of gallbladder contents from sludge to jelly-like appearance. All of gallbladder contents in this study were dried with bile and analyzed by IR spectroscopy. Generally, the components contained in bile are bile acid, cholesterol, lecithin, bilirubin and more, and do not contain volatile components [2]. Therefore, these analyses by IR spectroscopy were carried out in gallbladder contents which contained liquid components, and it was considered that only the water content of gallbladder contents was changed by drying. Moisture in bile is removed by the actions of the $\mathrm{Na}^{+}-\mathrm{K}^{+}$and $\mathrm{Cl}^{-}-\mathrm{HCO}_{3}{ }^{-}$pumps of gallbladder epithelial cells $[19,21]$ and/or-based on reports in mice-aquaporins, which are channels responsible for transporting water into the body $[18,24]$. These transport channels have been reported to possess the ability to concentrate bile or manipulate the composition of gallbladder bile. Therefore, abnormal functioning of bile moisture absorption mechanisms might be involved in the pathophysiology of canine biliary sludge and gallbladder mucoceles.

In this study, since the IR spectroscopy was a qualitative analysis method, it is necessary to quantitatively analyze components of gallbladder content, such as amount of mucin and/or cholesterol, in the future study. It is not known whether biliary sludge is similar to gallbladder mucoceles in terms of involvement of excessive mucin secretion from the gallbladder mucosal epithelium. Therefore, future studies on histopathological evaluation of gallbladder mucosal epithelium have been planned to investigate whether excessive mucin secretion is involved in the pathophysiology of biliary sludge. Additionally, in the case of gallbladder content transition with histological findings of severe gallbladder bleeding observed in the present study, it is necessary to histopathologically investigate the gallbladder and its principal components in detail.

To conclude, the present findings have demonstrated that the principle components of both biliary sludge and gallbladder mucoceles are mucins. These findings suggest the possibility that the two diseases are not independent of each other, but in fact, represent one continuous disease. Further studies involving extensive data collection of gallbladder contents from biliary sludge and gallbladder mucocele, and histopathological evaluation of these gallbladder will be conducted to elucidate the pathophysiologies of gallbladder mucoceles and biliary sludge.

\section{REFERENCES}

1. Besso, J. G., Wrigley, R. H., Gliatto, J. M. and Webster, C. R. 2000. Ultrasonographic appearance and clinical findings in 14 dogs with gallbladder mucocele. Vet. Radiol. Ultrasound 41: 261-271. [Medline] [CrossRef]

2. Center, S. A. 2009. Diseases of the gallbladder and biliary tree. Vet. Clin. North Am. Small Anim. Pract. 39: 543-598. [Medline] [CrossRef]

3. Center, S. A., Warner, K. L., McCabe, J., Foureman, P., Hoffmann, W. E. and Erb, H. N. 2005. Evaluation of the influence of S-adenosylmethionine on systemic and hepatic effects of prednisolone in dogs. Am. J. Vet. Res. 66: 330-341. [Medline] [CrossRef]

4. Center, S. A., Randolph, J. F., Warner, K. L., McCabe-McClelland, J., Foureman, P., Hoffmann, W. E. and Erb, H. N. 2005. The effects of S-adenosylmethionine on clinical pathology and redox potential in the red blood cell, liver, and bile of clinically normal cats. J. Vet. Intern. Med. 19: 303-314. [Medline] [CrossRef]

5. Choi, J., Kim, A., Keh, S., Oh, J., Kim, H. and Yoon, J. 2014. Comparison between ultrasonographic and clinical findings in 43 dogs with gallbladder mucoceles. Vet. Radiol. Ultrasound 55: 202-207. [Medline] [CrossRef]

6. Corfield, A. P. 2015. Mucins: a biologically relevant glycan barrier in mucosal protection. Biochim. Biophys. Acta 1850: 236-252. [Medline] 
[CrossRef]

7. Davis, C. P., Cleven, D., Balish, E. and Yale, C. E. 1977. Bacterial association in the gastrointestinal tract of beagle dogs. Appl. Environ. Microbiol. 34: 194-206. [Medline]

8. Festi, D., Montagnani, M., Azzaroli, F., Lodato, F., Mazzella, G., Roda, A., Di Biase, A. R., Roda, E., Simoni, P. and Colecchia, A. 2007. Clinical efficacy and effectiveness of ursodeoxycholic acid in cholestatic liver diseases. Curr. Clin. Pharmacol. 2: 155-177. [Medline] [CrossRef]

9. Gilad, R., Williams, J. C. Jr., Usman, K. D., Holland, R., Golan, S., Tor, R. and Lifshitz, D. 2016. Interpreting the results of chemical stone analysis in the era of modern stone analysis techniques. J. Nephrol. [CrossRef]. [Medline]

10. Goso, Y. 2016. Malonic acid suppresses mucin-type O-glycan degradation during hydrazine treatment of glycoproteins. Anal. Biochem. 496: 35-42. [Medline] [CrossRef]

11. Guarino, M. P., Cong, P., Cicala, M., Alloni, R., Carotti, S. and Behar, J. 2007. Ursodeoxycholic acid improves muscle contractility and inflammation in symptomatic gallbladders with cholesterol gallstones. Gut 56: 815-820. [Medline] [CrossRef]

12. Housset, C., Chrétien, Y., Debray, D. and Chignard, N. 2016. Functions of the Gallbladder. Compr. Physiol. 6: 1549-1577. [Medline] [CrossRef]

13. Iida, M., Okayama, Y., Goto, K., Shiraki, S., Hoshino, M. and Takeuchi, T. 1993. Gallstone classification and analysis of their constituents (in Japanese). Nippon Rinsho 51: 1718-1724. [Medline]

14. Jaswal, B. B., Kumar, V., Sharma, J., Rai, P. K., Gondal, M. A., Gondal, B. and Singh, V. K. 2016. Analysis of heterogeneous gallstones using laser-induced breakdown spectroscopy (LIBS) and wavelength dispersive X-ray fluorescence (WD-XRF). Lasers Med. Sci. 31: 573-579. [Medline] [CrossRef]

15. Kameda, H. 1993. The development of clinical studies in cholelithiasis. Intern. Med. 32: 193. [Medline] [CrossRef]

16. Krafft, C., Steiner, G., Beleites, C. and Salzer, R. 2009. Disease recognition by infrared and Raman spectroscopy. J. Biophotonics 2: 13-28. [Medline] [CrossRef]

17. Lawrence, Y. A., Ruaux, C. G., Nemanic, S. and Milovancev, M. 2015. Characterization, treatment, and outcome of bacterial cholecystitis and bactibilia in dogs. J. Am. Vet. Med. Assoc. 246: 982-989. [Medline] [CrossRef]

18. Li, L., Zhang, H., Ma, T. and Verkman, A. S. 2009. Very high aquaporin-1 facilitated water permeability in mouse gallbladder. Am. J. Physiol. Gastrointest. Liver Physiol. 296: G816-G822. [Medline] [CrossRef]

19. Meyer, G., Guizzardi, F., Rodighiero, S., Manfredi, R., Saino, S., Sironi, C., Garavaglia, M. L., Bazzini, C., Bottà, G., Portincasa, P., Calamita, G. and Paulmichl, M. 2005. Ion transport across the gallbladder epithelium. Curr. Drug Targets Immune Endocr. Metabol. Disord. 5: $143-151$. [Medline] [CrossRef]

20. Smalle, T. M., Cahalane, A. K. and Köster, L. S. 2015. Gallbladder mucocoele: A review. J. S. Afr. Vet. Assoc. 86: 1318 [CrossRef]. [Medline]

21. Swartz-Basile, D. A., Lu, D., Basile, D. P., Graewin, S. J., Al-Azzawi, H., Kiely, J. M., Mathur, A., Yancey, K. and Pitt, H. A. 2007. Leptin regulates gallbladder genes related to absorption and secretion. Am. J. Physiol. Gastrointest. Liver Physiol. 293: G84-G90. [Medline] [CrossRef]

22. Travo, A., Piot, O., Wolthuis, R., Gobinet, C., Manfait, M., Bara, J., Forgue-Lafitte, M. E. and Jeannesson, P. 2010. IR spectral imaging of secreted mucus: a promising new tool for the histopathological recognition of human colonic adenocarcinomas. Histopathology 56: 921-931. [Medline] [CrossRef]

23. Uno, T., Okamoto, K., Onaka, T., Fujita, K., Yamamura, H. and Sakai, T. 2009. Correlation between ultrasonographic imaging of the gallbladder and gallbladder content in eleven cholecystectomised dogs and their prognoses. J. Vet. Med. Sci. 71: 1295-1300. [Medline] [CrossRef]

24. van Erpecum, K. J., Wang, D. Q., Moschetta, A., Ferri, D., Svelto, M., Portincasa, P., Hendrickx, J. J., Schipper, M. and Calamita, G. 2006. Gallbladder histopathology during murine gallstone formation: relation to motility and concentrating function. J. Lipid Res. 47: 32-41. [Medline] [CrossRef]

25. Ward, A., Brogden, R. N., Heel, R. C., Speight, T. M. and Avery, G. S. 1984. Ursodeoxycholic acid: a review of its pharmacological properties and therapeutic efficacy. Drugs 27: 95-131. [Medline] [CrossRef]

26. Yoo, E. H., Oh, H. J. and Lee, S. Y. 2008. Gallstone analysis using Fourier transform infrared spectroscopy (FT-IR). Clin. Chem. Lab. Med. 46 : 376-381. [Medline] [CrossRef] 